\title{
Publisher Correction: A glycoprotein B-neutralizing antibody structure at $2.8 \AA$ uncovers a critical domain for herpesvirus fusion initiation
}

\author{
Stefan L. Oliver (1), Yi Xing, Dong-Hua Chen (D), Soung Hun Roh, Grigore D. Pintilie, David A. Bushnell (1), \\ Marvin H. Sommer, Edward Yang, Andrea Carfi, Wah Chiu (1) \& Ann M. Arvin (D)
}

Correction to: Nature Communications https://doi.org/10.1038/s41467-020-17911-0, published online 18 August 2020.

The original version of this Article contained errors in the author affiliations.

Yi Xing and Andrea Carfi were incorrectly associated with 'Division of Cryo-EM and Bioimaging SSRL, SLAC National Accelerator Laboratory, Menlo Park, CA 94025, USA' instead of the correct 'Syros Pharmaceuticals, Inc., Cambridge, MA 02140, USA' and 'Moderna, Cambridge, MA 02139, USA', respectively.

This has now been corrected in both the PDF and HTML versions of the Article.

Published online: 28 August 2020

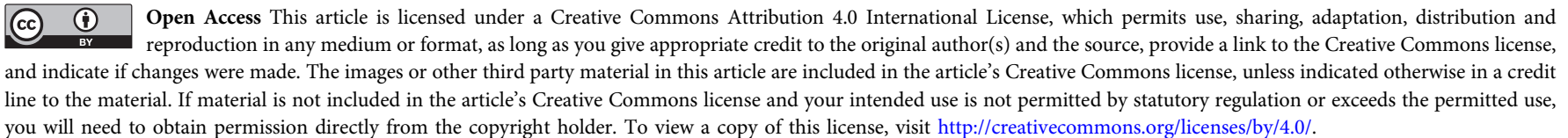

(c) The Author(s) 2020 